\title{
Scottish dental technicians: needs and status
}

\author{
Educational needs and employment status of Scottish dental technicians
}

M. K. Ross and R. J. Ibbetson. Br Dent J 2005; 198: 97-101

Objective To investigate the educational needs and employment status of dental technicians in Scotland.

Subjects Two hundred and fifty dental technicians with postal addresses in Scotland.

Design Structured questionnaire.

Results An 83\% response rate was achieved following three mailings. The majority of respondents were employed in commercial dental laboratories largely within the 'central belt' of Scotland, with 96\% stating they were in full-time employment. Only 33\% of these essential health-care workers were voluntarily registered with the Dental Technicians' Association, suggesting that a significant number had not felt it necessary or beneficial to do so. A lack of educational structure was identified, as was poor remuneration and an absence of opportunity for career progression. Although the prospect of continuing professional development was desirable, many respondents reported that they would be penalised finanically for undertaking this and, in addition, may not be given the opportunity to pursue education because of lack of co-operation from their employer. Only $47 \%$ had attended an educational event within the preceding year, and of those who had not done this, a period of two-32 years had elapsed since any CPD involvement. Of the respondents, only $34 \%$ stated that any financial assistance had been available for educational purposes, with access to education being highlighted as problematic by $68 \%$. A total of $64 \%$ of subjects felt they were out-of-date with professional education.

Conclusions This study highlights a number of real and potential problems in the field of education in dental technology. It is apparent that change within the structure of education and professional status, although largely welcomed, may be difficult to implement. The profession, as a whole, must realise that these changes in education and employment are not optional, and should be embraced as a positive step which will hopefully raise the profile and status of dental technicians throughout the UK.

\section{IN BRIEF}

- The education and training system for dental technicians in Scotland currently lacks resource and structure.

- The advent of mandatory CPD following registration will be difficult for many people in Scotland to access because of geographical location.

- The shortage of funding for CPD poses a problem for dental technicians.

- Rates of remuneration are inadequate and unlikely to encourage people into the profession in the future.

\section{COMMENT}

Dental technician training and education, and indeed, arguably, dental technology, are in crisis.

This paper, which is very timely, indicates the extent of the problems of dental technician education, retention and working arrangements that apply in Scotland. It is however, unlikely that the situation is much different in other parts of the UK.

Among the greatest causes for concern are the findings that $64 \%$ felt that they were not up to date with their education in dental technology, the comments about long hours for poor rewards, and the intention of some to leave dental technology.

The GDC's plans for registration of dental technicians will hopefully improve the situation in the long term but only if the educational arrangements, status and financial rewards for this important group of dental professionals are significantly improved. In the short term however the recently announced GDC decision that 'registration should be compulsory for everyone directly involved in the manufacture of dental appliances', is likely to make more difficulty in the medium term. This will particularly be the case at the end of the two-year transitional period after which only qualified dental technicians will be able to register.

The proposed intention of the Scottish Executive that there should be at least 15-20 dental technicians qualifying annually in Scotland is welcomed. However, it is essential that this proposal is brought to fruition in the immediate future because it takes time from the appointment of trainee technicians to the time they contribute to the qualified technician workforce. Similar developments, with appropriate resources, are required throughout the UK.

The authors state that 'The entire structure of education and career progression in dental technology should be addressed as a matter of urgency'. In my view this is long overdue and must be undertaken now. The consequences for dentistry and our patients of not doing so will be profound, particularly with the need for high quality restorations in removable prosthodontics, crown and bridgework, implantology and maxillofacial prosthodontics.

It is not only dental technicians that need to be alerted to the forthcoming registration of dental technicians; dentists, the government, employing authorities and our patients should be aware of these changes. The potential problems which will arise if these changes are not managed in an appropriate way and the status, initial and continuing education arrangements and working conditions of dental technicians are not significantly improved should not be underestimated.

I. B. Watson, Consultant in Restorative Dentistry, Glasgow Dental Hospital and School

doi: 10.1038/sj.bdj.4812531 